\title{
Recent progress in some exclusive and semi-exclusive pro- cesses in proton-proton collisions
}

\author{
Antoni Szczurek ${ }^{1,2, a}$, Anna Cisek ${ }^{2, b}$, Marta Łuszczak ${ }^{2, c}$, and Wolfgang Schäfer ${ }^{1, d}$ \\ ${ }^{1}$ Institute of Nuclear Physics, Kraków, ul. Radzikowskiego 152 \\ ${ }^{2}$ Rzeszòw University, Rzeszów, ul. Rejtana 16
}

\begin{abstract}
We present the main results of our recent analyses of exclusive production of vector charmonia $\left(J / \psi\right.$ and $\left.\psi^{\prime}\right)$ in $k_{t}$-factorization approach and for $\gamma \gamma$ production of charged dilepton pairs in exclusive and semiinclusive processes in a new approach, similar in spirit to $k_{t}$-factorization.

The results for charmonia are compared with recent results of the LHCb collaboration. We include some helicity flip contributions and quantify the effect of absorption correction. The effect of $c \bar{c}$ wave function is illustrated.

We present uncertainties related to $F_{2}$ structure function which are the main ingredient of the approach. Our results are compared with recent CMS data for dilepton production with lepton isolation cuts imposed.
\end{abstract}

\section{Introduction}

Here we briefly summarize our recent results for exclusive production of $J / \psi$ mesons obtained in $[1,2]$ and for photon-photon production of charged dileptons obtained in $[5,6]$.

\section{Exclusive production of vector charmonia}

The Born diagrams for the exclusive production of $J / \psi$ mesons are shown in Fig.1. In actual calculations in $[1,2]$ we include also absorption effects due to soft proton-proton interactions.

All details of the formalism can be found in Refs.[1] and [2]. Here we only sketch the main points. Imaginary part of the forward $\gamma p \rightarrow J / \psi p$ amplitude

$$
\begin{array}{r}
\mathfrak{J} m \mathcal{M}_{T}\left(W, \Delta^{2}=0, Q^{2}=0\right)=W^{2} \frac{c_{v} \sqrt{4 \pi \alpha_{e m}}}{4 \pi^{2}} 2 \int_{0}^{1} \frac{d z}{z(1-z)} \int_{0}^{\infty} \pi d k^{2} \psi_{V}\left(z, k^{2}\right) \\
\int_{0}^{\infty} \frac{\pi d \kappa^{2}}{\kappa^{4}} \alpha_{S}\left(q^{2}\right) \mathcal{F}\left(x_{\mathrm{eff}}, \kappa^{2}\right)\left(A_{0}\left(z, k^{2}\right) W_{0}\left(k^{2}, \kappa^{2}\right)+A_{1}\left(z, k^{2}\right) W_{1}\left(k^{2}, \kappa^{2}\right)\right) .
\end{array}
$$

\footnotetext{
a e-mail: antoni.szczurek@ifj.edu.pl

be-mail: acisek@univ.rzeszow.pl

ce-mail: luszczak@univ.rzeszow.pl

de-mail: Wolfgang.Schafer@ifj.edu.pl
} 

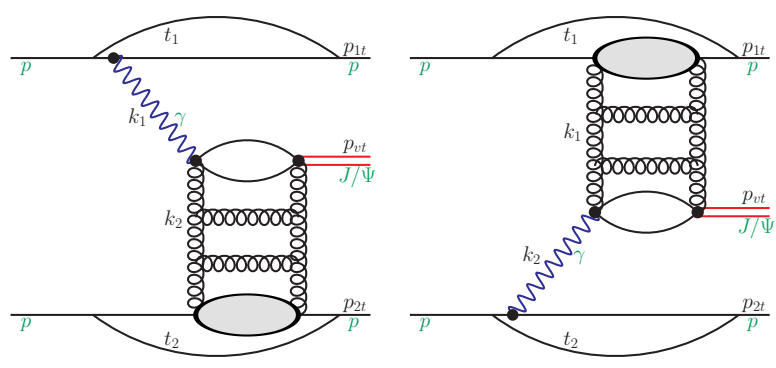

Figure 1. Born diagrams for exclusive production of $J / \psi$ mesons.

The full amplitude, at finite momentum transfer is parametrized as:

$$
\mathcal{M}\left(W, \Delta^{2}\right)=(i+\rho) \mathfrak{J} m \mathcal{M}\left(W, \Delta^{2}=0, Q^{2}=0\right) \exp \left(-B(W) \Delta^{2} / 2\right),
$$

Then the amplitude for the $p p \rightarrow p p J / \psi$ can be written somewhat formally as:

$$
\begin{array}{r}
\mathcal{M}_{h_{1} h_{2} \rightarrow h_{1} h_{2} V}^{\lambda_{1} \lambda_{2} \rightarrow \lambda_{1}^{\prime} \lambda_{2}^{\prime} \lambda_{V}}\left(s, s_{1}, s_{2}, t_{1}, t_{2}\right)=\mathcal{M}_{\gamma \mathbf{P}}+\mathcal{M}_{\mathbb{P} \gamma} \\
=\left\langle p_{1}^{\prime}, \lambda_{1}^{\prime}\left|J_{\mu}\right| p_{1}, \lambda_{1}\right\rangle \epsilon_{\mu}^{*}\left(q_{1}, \lambda_{V}\right) \frac{\sqrt{4 \pi \alpha_{e m}}}{t_{1}} \mathcal{M}_{\gamma^{*} h_{2} \rightarrow V h_{2}}^{\lambda_{\gamma^{*} \lambda_{2} \rightarrow \lambda_{V} \lambda_{2}}}\left(s_{2}, t_{2}, Q_{1}^{2}\right) \\
+\left\langle p_{2}^{\prime}, \lambda_{2}^{\prime}\left|J_{\mu}\right| p_{2}, \lambda_{2}\right\rangle \epsilon_{\mu}^{*}\left(q_{2}, \lambda_{V}\right) \frac{\sqrt{4 \pi \alpha_{e m}}}{t_{2}} \mathcal{M}_{\gamma^{*} h_{1} \rightarrow V h_{1}}^{\lambda_{\gamma^{*} \lambda_{1} \rightarrow \lambda_{V} \lambda_{1}}}\left(s_{1}, t_{1}, Q_{2}^{2}\right) .
\end{array}
$$

The auxiliary amplitude in Eq. (3) for the emission of a photon of transverse polarization $\lambda_{V}$, and transverse momentum $\boldsymbol{q}_{1}=-\boldsymbol{p}_{1}$ can be written as:

$$
\left\langle p_{1}^{\prime}, \lambda_{1}^{\prime}\left|J_{\mu}\right| p_{1}, \lambda_{1}\right\rangle \epsilon_{\mu}^{*}\left(q_{1}, \lambda_{V}\right)=\frac{\left(\boldsymbol{e}^{*\left(\lambda_{V}\right)} \boldsymbol{q}_{1}\right)}{\sqrt{1-z_{1}}} \frac{2}{z_{1}} \chi_{\lambda^{\prime}}^{\dagger}\left\{F_{1}\left(Q_{1}^{2}\right)-\frac{i \kappa_{p} F_{2}\left(Q_{1}^{2}\right)}{2 m_{p}}\left(\boldsymbol{\sigma}_{1} \cdot\left[\boldsymbol{q}_{1}, \boldsymbol{n}\right]\right)\right\} \chi_{\lambda} .
$$

Above $F_{1}$ and $F_{2}$ are Dirac and Pauli electromagnetic form factors, respectively. In Ref.[2] we have included the Pauli form factors for a first time.

\section{Selected results for vector quarkonia}

Rapidity distributions of $J / \psi$ mesons are show in Fig.2. In this calculation Gaussian wave functions were used. Only results with UGDFs that include nonlinear effects describe the LHCb experimental distributions [3]. In our opinion it is too preliminary to conclude that we observe nonlinear effects or onset of gluon saturation. In Ref.[2] we obtained similar distributions for $\psi^{\prime}$ [2].

Very interesting quantity is the ratio of the cross sections for $\psi^{\prime}$ and $J / \psi$ production. As shown in Fig. 3 such a ratio is very sensitive to the functional form of the $c \bar{c}$ light-cone wave function. We conclude that the Gauss $c \bar{c}$ wave function much better describes the LHCb data [3] than Coulomb WF. In some calculation in the literature the wave functions are not included explicitly and only point-like coupling is used [4]. 

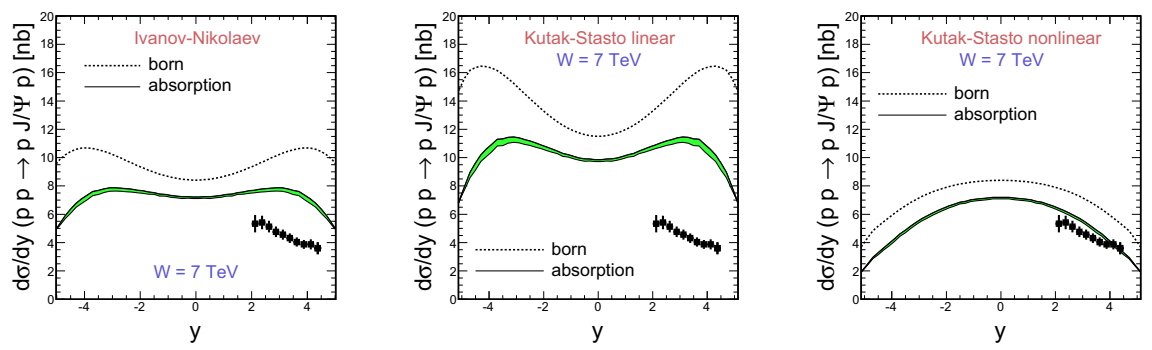

Figure 2. Rapidity distributions for three different unintegrated gluon distributions. The upper curves were obtained in the Born approximation and the lower band includes absorption effects.
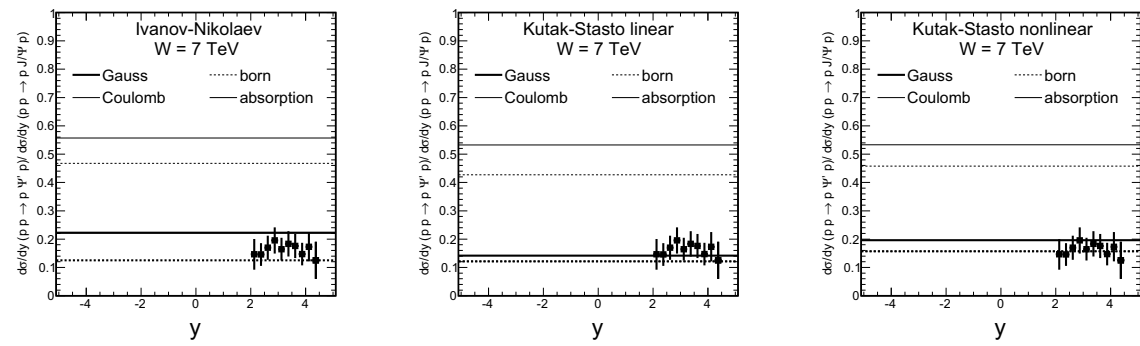

Figure 3. The ratio of the cross section for $\psi^{\prime}$ and $J / \psi$.
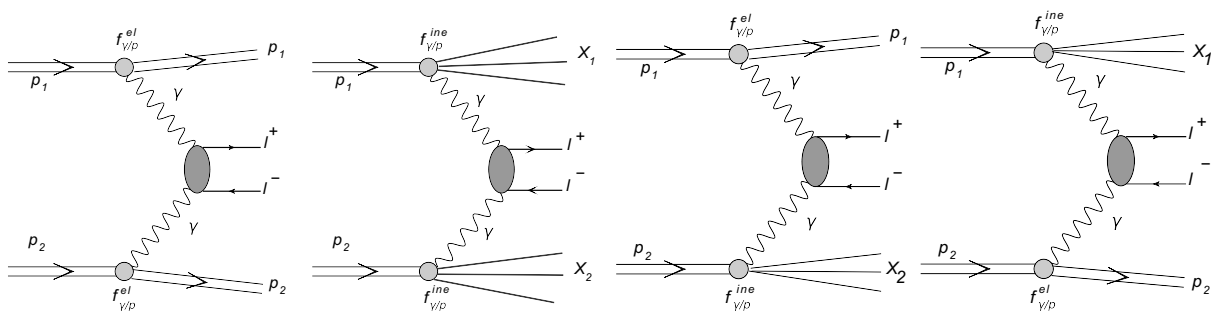

Figure 4. The different categories of $\gamma \gamma$ processes.

\section{$4 \gamma \gamma$ production of dileptons}

The $\gamma \gamma$ processes can be categorize according to the final state (see Fig.4). All the processes were discussed in $[5,6]$.

Two different approaches were discussed in the context of $\gamma \gamma$ production of dileptons. 
In the collinear approach the corresponding cross sections are calculated respectively as:

$$
\frac{d \sigma^{(k, l)}}{d y_{1} d y_{2} d^{2} p_{t}}=\frac{1}{16 \pi^{2} \hat{s}^{2}} x_{1} \gamma_{k}\left(x_{1}, \mu^{2}\right) x_{2} \gamma_{l}\left(x_{2}, \mu^{2}\right) \overline{\left|\mathcal{M}_{\gamma \gamma \rightarrow l^{+} l^{-}}\right|^{2}},
$$

where $k, l \in\{\mathrm{el}, \mathrm{in}\}$ stand for the processes with intact (el) or dissociated in proton at the photon vertex. The $\gamma_{k}\left(x, \mu^{2}\right)$ are the corresponding photon distributions in a proton, with or without the condition of breakup. The elastic photon distributions are calculated with the help of the nucleon electromagnetic form factors, while the inelastic distributions can be obtained by using a combined QCD/QED evolution [7].

In the $k_{t}$-factorization, proposed recently in $[5,6]$, the fully unintegrated photon flux can be written as (below incoming particles are denoted as $A, B$ ):

$$
\frac{d \mathcal{F}_{\gamma^{*} \leftarrow A}\left(z, \boldsymbol{q}, M_{X}^{2}\right)}{d M_{X}^{2}}=\frac{\alpha_{\mathrm{em}}}{\pi}(1-z)\left(\frac{\boldsymbol{q}^{2}}{\boldsymbol{q}^{2}+z\left(M_{X}^{2}-m_{A}^{2}\right)+z^{2} m_{A}^{2}}\right)^{2} \cdot \frac{p_{B}^{\mu} p_{B}^{v}}{s^{2}} W_{\mu v}\left(M_{X}^{2}, Q^{2}\right) .
$$

Information on the virtual photon-proton interaction is contained in the hadronic tensor, which is obtained in terms of the electromagnetic currents as:

$$
W_{\mu \nu}\left(M_{X}^{2}, Q^{2}\right)=\overline{\sum_{X}}(2 \pi)^{3} \delta^{(4)}\left(p_{X}-p_{A}-q\right)\left\langle p\left|J_{\mu}\right| X\right\rangle\left\langle X\left|J_{v}^{\dagger}\right| p\right\rangle d \Phi_{X}
$$

The virtual photoabsorption cross sections are related to hadronic tensors as:

$$
\sigma_{T}\left(\gamma^{*} p\right)=\frac{4 \pi \alpha_{e m}}{4 \sqrt{X}}\left(-\frac{\delta_{\mu v}^{\perp}}{2}\right) 2 \pi W^{\mu v}\left(M_{X}^{2}, Q^{2}\right), \sigma_{L}\left(\gamma^{*} p\right)=\frac{4 \pi \alpha_{e m}}{4 \sqrt{X}} e_{\mu}^{0} e_{v}^{0} 2 \pi W^{\mu v}\left(M_{X}^{2}, Q^{2}\right)
$$

It is customary to introduce dimensionless structure function $F_{i}\left(x_{\mathrm{Bj}}, Q^{2}\right), i=T, L$ as

$$
\sigma_{T, L}\left(\gamma^{*} p\right)=\frac{4 \pi^{2} \alpha_{e m}}{Q^{2}} \frac{1}{\sqrt{1+\frac{4 x_{\mathrm{Bj}}^{2} m_{A}^{2}}{Q^{2}}}} F_{T, L}\left(x_{\mathrm{Bj}}, Q^{2}\right),
$$

At high energies, in the calculation of photon fluxes, the contribution of the structure function

$$
F_{2}\left(x_{\mathrm{Bj}}, Q^{2}\right)=\frac{F_{T}\left(x_{\mathrm{Bj}}, Q^{2}\right)+F_{L}\left(x_{\mathrm{Bj}}, Q^{2}\right)}{1+\frac{4 x_{\mathrm{Bj}}^{2} m_{A}^{2}}{Q^{2}}}
$$

dominates.

The unintegrated fluxes enter the cross section for dilepton production as

$$
\frac{d \sigma^{(i, j)}}{d y_{1} d y_{2} d^{2} \boldsymbol{p}_{1} d^{2} \boldsymbol{p}_{2}}=\int \frac{d^{2} \boldsymbol{q}_{1}}{\pi \boldsymbol{q}_{1}^{2}} \frac{d^{2} \boldsymbol{q}_{2}}{\pi \boldsymbol{q}_{2}^{2}} \mathcal{F}_{\gamma^{*} / A}^{(i)}\left(x_{1}, \boldsymbol{q}_{1}\right) \mathcal{F}_{\gamma^{*} / B}^{(j)}\left(x_{2}, \boldsymbol{q}_{2}\right) \frac{d \sigma^{*}\left(p_{1}, p_{2} ; \boldsymbol{q}_{1}, \boldsymbol{q}_{2}\right)}{d y_{1} d y_{2} d^{2} \boldsymbol{p}_{1} d^{2} \boldsymbol{p}_{2}},
$$

where $i, j \in\{\mathrm{el}, \mathrm{in}\}$ again refer to elastic and inelastic processes on the proton sides. For brevity we integrated over invariant masses of the possible dissociated system.

The longitudinal momentum fractions carried by photons can be obtained from rapidities and transverse momenta of the charged leptons as:

$$
x_{1}=\sqrt{\frac{\boldsymbol{p}_{1}^{2}+m_{l}^{2}}{s}} e^{y_{1}}+\sqrt{\frac{\boldsymbol{p}_{2}^{2}+m_{l}^{2}}{s}} e^{y_{2}}, x_{2}=\sqrt{\frac{\boldsymbol{p}_{1}^{2}+m_{l}^{2}}{s}} e^{-y_{1}}+\sqrt{\frac{\boldsymbol{p}_{2}^{2}+m_{l}^{2}}{s}} e^{-y_{2}} .
$$



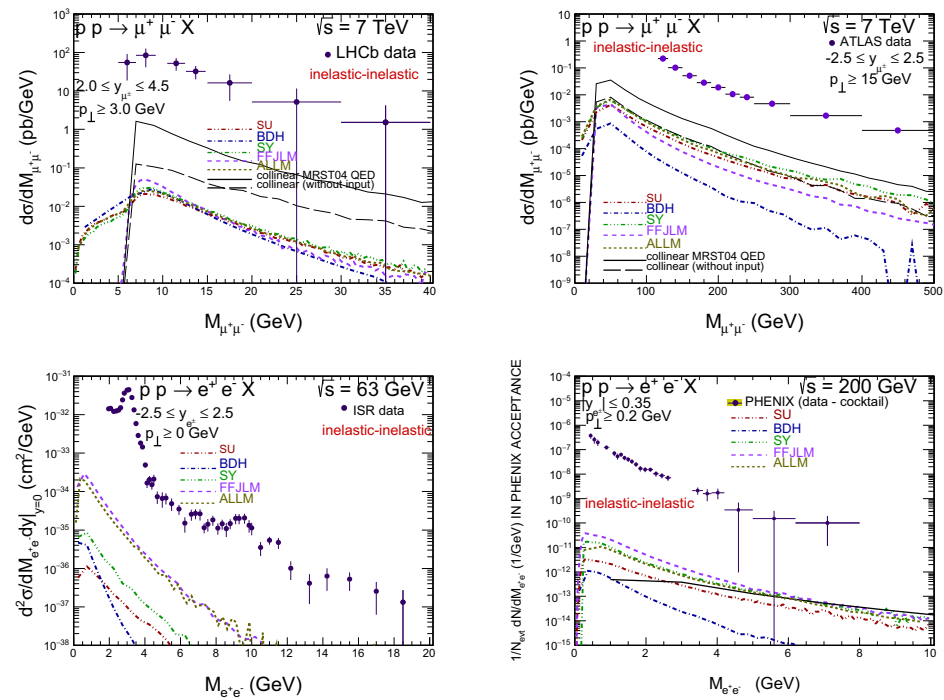

Figure 5. Dilepton invariant mass distributions for different experimental conditions (see the four panels) and for different structure functions (see different lines).

\section{Selected results for $l^{+} l^{-}$production}

As an example in Fig.5 we show invariant mass distributions for double dissociative processes (both protons undergo electromagnetic dissociation). The calculations have been performed for different experimental conditions specified in the figure legend. We show results for different proton structure functions from the literature. The different structure functions lead to quite different results. The presented results strongly depend on the kinematical regions of longitudinal momentum fraction and photon virtuality $\left(x, Q^{2}\right)$ which were not sufficiently well studied experimentally and theoretically in which an interplay of perturbative and nonperturbative effects takes place. We observe that the $\gamma \gamma$ contribution constitutes only a small fraction of the cross section compared to the experimental data and Drell-Yan contribution (not shown explicitly here). Similar results for elastic-inelastic channel were shown in [5].

Now we wish to show results which can be directly compared to experimental data [8]. The data were obtained by imposing a kinematical constraint on lepton isolation. The results are shown in Fig.6 A relatively good agreement has been achieved. A better quality results are expected from Run II at the LHC.

\section{Conclusions}

Our results and open problems for charmonia production can be summarized as follows. We have found some model dependent indication of presence of nonlinear effects in the small- $x$ gluon distribution of a proton. It is important to remember, that the present experiments are not fully exclusive and rather semi-inclusive processes have to be studied. Experimentally so far proton dissociation has been "extracted" in a model dependent way assuming some functional form in $p_{t}$. From HERA we 

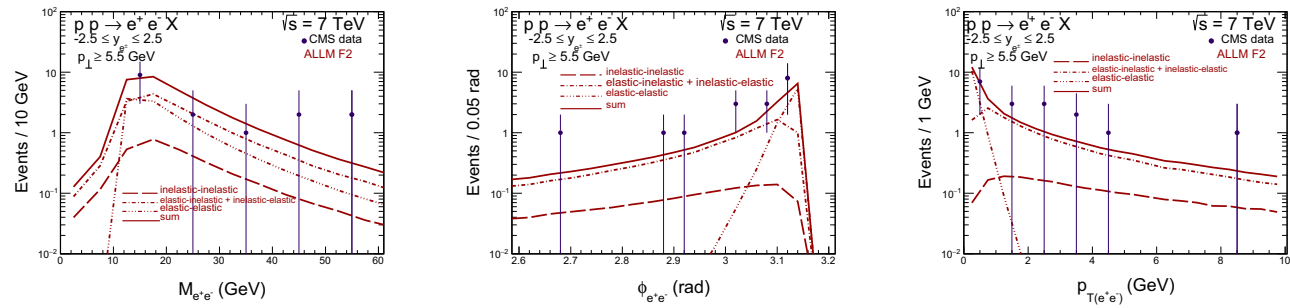

Figure 6. Dilepton invariant mass distributions (left) distribution in relative azimuthal angle between leptons (middle) and distribution in transverse momentum of the pair of leptons (right) for a selected structure function.

have some limited knowledge only about diffractive dissociation. Compared to exclusive production of $J / \psi$ at HERA, in $p p$ collisions there is also electromagnetic dissociation. Interference effects due to the two diagrams in Fig. 1 were predicted. It would be nice to see a modulation in $\phi_{p p}$ due to interference effects between the two diagrams. In the future, the CMS+TOTEM and ATLAS+ALFA experiments could measure purely exclusive reaction and study dependence on many more variables.

Our results for photon-photon induced processes can be summarized as: We discussed two different approaches for $\gamma \gamma$ processes - collinear and $k_{t}$-factorization, they are not completely equivalent. We have found strong dependence on the structure function input to the photon fluxes in the $k_{t}$-factorization approach. Semi-exclusive contributions with dissociation are large which is an interesting lesson for $p p \rightarrow p p J / \psi$. The photon-photon contributions are, however, rather small compared to the Drell-Yan contribution. A reasonable description of the CMS data with isolated electrons was obtained (recently also the ATLAS collaboration obtained similar results). So far only the collinear approach has been applied e.g. to the $p p \rightarrow(\gamma \gamma) \rightarrow W^{+} W^{-} X Y$ processes. Such a reaction is important in searches for Beyond Standard Model effects. A large cross section was found [9]. A calculation in $k_{t}$-factorization approach would be very valuable.

\section{References}

[1] W. Schäfer and A. Szczurek, Phys. Rev. D76 (2007) 094014.

[2] A. Cisek, W. Schäfer and A. Szczurek, JHEP 1504 (2015) 159,

[3] R. Aaij et al. [LHCb Collaboration], J. Phys. G 40 (2013) 045001 [arXiv:1301.7084 [hep-ex]]; R. Aaij et al. [LHCb Collaboration], J. Phys. G 41 (2014) 055002 [arXiv:1401.3288 [hep-ex]].

[4] S. P. Jones, A. D. Martin, M. G. Ryskin and T. Teubner, JHEP 1311 (2013) 085 [arXiv:1307.7099].

[5] M. Łuszczak, W. Schäfer and A. Szczurek, arXiv:1510.00294,

[6] G. Gil da Silveira, L. Forthomme, K. Piotrzkowski, W. Schäfer, A. Szczurek, JHEP 1502 (2015) 159 ,

[7] A. D. Martin, R. G. Roberts, W. J. Stirling and R. S. Thorne, Eur. Phys. J. C 39, 155 (2005) [hep-ph/0411040].

[8] S. Chatrchyan et al. [CMS Collaboration], JHEP 1211, 080 (2012) [arXiv:1209.1666 [hep-ex]].

[9] M. Łuszczak, A. Szczurek and C. Royon, JHEP 1502 (2015) 098 [arXiv:1409.1803 [hep-ph]]. 